\title{
Moderately Hypofractionated Radiotherapy Alone for Stage I-IIB Non-small Cell Lung Cancer
}

Ryan T. Hughes ${ }^{1}$, Corbin A. Helis ${ }^{1}$, Michael H. Soike ${ }^{1}$, Beverly J. Levine ${ }^{2}$, Michael Farris ${ }^{1}$, Arthur W. Blackstock ${ }^{1}$

1. Radiation Oncology, Wake Forest School of Medicine, Winston-Salem, USA 2. Epidemiology, Wake Forest School of Medicine, Winston-Salem, USA

Corresponding author: Michael Farris, mfarris@wakehealth.edu

\begin{abstract}
Introduction: The optimal management of patients with early non-small cell lung cancer (NSCLC) not amenable to surgical resection or stereotactic body radiotherapy (SBRT) or those with hilar nodal involvement ineligible for surgery or concurrent chemoradiotherapy is unclear. This report describes survival outcomes and toxicity profiles of patients treated with hypofractionated radiotherapy (HRT) alone.

Methods: A total of 52 patients with Stage I-IIB NSCLC treated with HRT alone between 2010-2018 were reviewed. Patients were categorized as having ultracentral tumors if the planning target volume contacted or overlapped the proximal bronchial tree, esophagus, pulmonary vein or artery. Overall survival (OS) and progression-free survival (PFS) were estimated using the Kaplan-Meier method and the competing risk cumulative incidence of locoregional failure (LRF) and distant failure (DF) were estimated using death without failure as a competing risk. Pneumonitis and esophagitis rates were evaluated as per Acute Common Terminology Criteria for Adverse Events (CTCAE) version 4.0.
\end{abstract}

Results: Of the 52 patients analyzed, 50 patients were treated with radiotherapy alone to a dose of 70.2 Gy in 26 fractions, one patient was treated with 68 Gy in 25 fractions and one patient was treated with 65 Gy in 26 fractions. The median age was 72 (range 48-89), $42 \%$ of patients had an Eastern Cooperative Oncology Group (ECOG) performance status of $2-3,46 \%$ were stage I and $54 \%$ were stage II. Hilar nodal involvement was present in $21 \%$ of patients and $74 \%$ of node-negative patients had ultracentral primary tumors. Median OS was 39.6 months and the median PFS was 21.0 months. Overall three-year cumulative incidence of LRF and DF were $32 \%$ and $34 \%$, respectively. Grade 3 pneumonitis occurred in two (4\%) patients. No grade 3+ acute esophagitis or grade $4-5$ toxicities were observed.

Conclusion: Hypofractionated thoracic radiotherapy consisting of $70.2 \mathrm{~Gy}$ is well-tolerated and results in favorable locoregional control for stage I-IIB patients who are not candidates for SBRT, surgery, or concurrent chemoradiotherapy.

Received 05/30/2019

Review began 06/04/2019 Review ended 06/19/2019 Published 06/22/2019

๑) Copyright 2019 Hughes et al. This is an open access article distributed under the terms of the Creative Commons Attribution License CC-BY 3.0., which permits unrestricted use, distribution, and reproduction in any medium, provided the original author and source are credited.
Categories: Radiation Oncology, Oncology

Keywords: hypofractionated radiation therapy, non-small cell lung cancer, ultracentral, medically inoperable, surgically inoperable

\section{Introduction}

Patients with operable, early stage non-small cell lung cancer (NSCLC) may be appropriately managed with either surgical resection or stereotactic body radiotherapy (SBRT). For inoperable patients or those who decline surgery, SBRT offers excellent local control and limited toxicity [1-2]. However, patients with tumors larger than $5 \mathrm{~cm}$, those within $2 \mathrm{~cm}$ of the proximal bronchial tree or overlapping mediastinal structures (ultracentral) are at increased risk for treatment-related morbidity after SBRT if normal tissue constraints cannot be achieved [3-5]. Various hypofractionated radiotherapy (HRT) regimens have been reported for the management of these patients [6-8], including the Cancer and Leukemia Group B (CALGB) 39904 study [9]. This phase I study evaluated various HRT regimens to a total dose of $70 \mathrm{~Gy}$ in 17-29 fractions without concurrent chemotherapy for early stage T1-T2N0 NSCLC. Disease control was favorable and no doselimiting toxicity was observed.

The non-surgical management of these patients with stage I-IIB NSCLC not amenable to SBRT can be challenging. Various definitive radiotherapeutic regimens have been reported, though no clear standard exists [10]. Within the SBRT literature, dose-escalation to an approximate biologically effective dose (BED) 10 of 100 Gy may improve local control [11-12]. Emerging data in the context of locally advanced NSCLC provide a rationale for dose-escalation using altered fractionation with or without concurrent or sequential chemotherapy [13-14]. The BED10 of these regimens ranges from 58.5-78 Gy.

Current guidelines recommend concurrent chemoradiotherapy followed by consolidative immunotherapy for patients with stage II NSCLC with hilar involvement [15]. However, various comorbidities, medical 
contraindications or patient preference sometimes preclude the delivery of concurrent chemotherapy. In these cases, radiotherapy alone is often utilized. Multiple studies have employed various altered fractionation regimens in the management of NSCLC patients with radiotherapy alone and a benefit to survival outcomes has been suggested with the use of modified fractionation compared to conventional fractionation [16]. In patients with medically inoperable disease that are not candidates for chemotherapy, 45 Gy delivered in 15 fractions (BED10 = 58.5 Gy) is a commonly utilized HRT schedule with a favorable toxicity profile and locoregional tumor control rates between $58 \%$ and $70 \%$, similar to that of conventionally-fractionated RT alone [17-18]. However, stage II patients were not well-represented in these two studies which also reported distant failure in approximately $40 \%-46 \%$.

At our institution, patients with stage I-IIB NSCLC who were ineligible for either resection, SBRT or combined chemoradiotherapy were treated with moderately hypofractionated radiotherapy alone based on CALGB 39904. The most common HRT regimen utilized was 70.2 Gy in 26 fractions (BED10 = 89.15 Gy) [19]. We aimed to evaluate the tumor control, survival outcomes, and toxicity profile of this regimen.

\section{Materials And Methods \\ Patient population, evaluation, and treatment}

In an Institutional Review Board-approved retrospective analysis, we reviewed the records of 92 patients with NSCLC receiving curative-intent HRT at the Wake Forest University Comprehensive Cancer Center between January of 2010 and June of 2018. Study data were collected and managed using research electronic data capture (REDCap) tools hosted at the Wake Forest School of Medicine Clinical and Translational Science Institute [20]. We identified 52 patients with newly-diagnosed American Joint Committee on Cancer 7th Edition Stage I-IIB NSCLC available for analysis. All patients underwent multidisciplinary thoracic oncology evaluation and all underwent staging imaging using [18F]fluorodeoxyglucose positron emission tomography. Brain magnetic resonance imaging (MRI) was employed in 13 of 24 stage I patients and 15 of 28 stage II patients. Patients were thoroughly counseled regarding the potential treatment options including surgery, radiotherapy, chemotherapy, or a combination. Those who were surgical candidates but declined surgery were offered radiotherapy. Treatment volumes were generated using four-dimensional computed tomography (CT) simulation as previously described [19]. The dose regimen prescribed was determined at the discretion of the treating physician when considering the target and normal tissues at risk on an individual basis. Per institutional preference, the majority of patients (96\%) were treated with 70.2 Gy in 26 fractions using 3D conformal radiotherapy (3D-CRT), intensity-modulated radiotherapy (IMRT) or volumetric modulated arc therapy (VMAT). Elective nodal irradiation was not performed. Post-treatment systemic therapy was not routinely utilized.

Available treatment plans were reviewed to define the location of the primary tumor, its proximity to central structures, as well as dosimetric factors. The primary tumor size was defined by its greatest diameter (in $\mathrm{cm}$ ) in any dimension. Dosimetric indices that were collected included the volume of the planning target volume (PTV), percentage of bilateral lung receiving 5, 20, 30 and $40 \mathrm{~Gy}$ (V5, V20, V30, and V40) and esophagus maximum dose were abstracted from the radiotherapy treatment planning system. Given the retrospective nature of this study and the inconsistency of documentation of cardiac outcomes, heart dosimetric parameters were not collected. Patients were categorized as having ultracentral tumors if the PTV contacted or overlapped the proximal bronchial tree, esophagus, pulmonary vein or artery, as previously defined [8]. Due to the inconsistency in the definition of ultracentral lung tumors, we chose not to deviate from this definition which does not include the heart or pericardium $[5,8]$. The proximal bronchial tree was defined according to the Radiation Therapy Oncology Group (RTOG) guidelines as the central airway from the distal trachea to the branching of the lobar bronchi [21].

\section{Outcomes}

Outcome measures were estimated from the date of completion of radiotherapy. Local failure (LF) was defined as either biopsy-proven disease or any radiographic evidence of recurrence at the treated primary site after completion of therapy that was recognized as such within the medical record. Regional failure was defined as the development of pathologic or radiographic evidence of disease in the regional lymph node stations. Locoregional failure (LRF) represents the occurrence of either local failure, regional failure, or both. Distant failure (DF) was defined as any pathologic or radiographic evidence of distant metastases. Overall survival was defined as the duration of time from completion of HRT to death from any cause or last follow-up. Progression-free survival was defined as the duration of time from completion of HRT to any progression event (local, regional, and/or distant failure), death from any cause, or last follow-up. Lung and esophageal toxicity were graded per the Common Terminology Criteria for Adverse Events (CTCAE) version 4.0 based upon review of the medical record [22]. Other toxicity data collected included objective measures such as steroid use for respiratory symptoms within 90 days (whether or not this was attributed to pneumonitis versus an unrelated indication) and any esophageal toxicity necessitating dilation, instrumentation, or feeding tube support.

\section{Statistical analysis}

Data were summarized using count (frequency) for categorical and median (range) for continuous variables. 


\section{Cureus}

Time to event analyses were performed from the date of completion of radiotherapy. Duration of follow-up was estimated using the reverse Kaplan-Meier method. OS and PFS were estimated using the Kaplan-Meier method and compared using the log-rank test. Cumulative incidence (CI) of LF, RF, DF, and LRF were estimated using competing risk methodology with death without failure as the competing risk and compared using Gray's test. Lung and esophageal dosimetric parameters were evaluated for association with pneumonitis and esophagitis using bivariate logistic regression and the Mann-Whitney U test. Cases with missing data for a given variable were excluded from analysis. All statistical analyses were performed using $\mathrm{R}$ version 3.5 (R Foundation for Statistical Computing, Vienna, Austria).

\section{Results}

\section{Study population}

Patient and disease characteristics are described in Table 1 . The majority of patients (58\%) had good performance status (Eastern Cooperative Oncology Group (ECOG) 0-1) and most were former smokers (82\%). T1-2 tumors were most common (83\%) and 21\% had N1 disease. Of 52 patients, 39 (75\%) had either ultracentral primary tumors or hilar lymph node involvement. Reasons the patients did not undergo surgery included the following: surgically unresectable disease, medically inoperable patient, both, or patient declined surgery.

\begin{tabular}{|c|c|}
\hline & Value $(n=52)$ \\
\hline Age at Diagnosis (years), median (range) & $72(48-89)$ \\
\hline \multicolumn{2}{|l|}{ ECOG Performance Status, n (\%) } \\
\hline 0 & $4(8)$ \\
\hline 1 & $26(50)$ \\
\hline 2 & $14(27)$ \\
\hline 3 & $8(15)$ \\
\hline \multicolumn{2}{|l|}{ Histology, n (\%) } \\
\hline Adenocarcinoma & $16(31)$ \\
\hline Squamous cell carcinoma & $26(50)$ \\
\hline NSCLC NOS & $6(12)$ \\
\hline Not biopsied & $4(8)$ \\
\hline \multicolumn{2}{|l|}{ Smoking Status, n (\%) } \\
\hline Current smoker & $9(18)$ \\
\hline Former smoker & $42(82)$ \\
\hline Smoking History (pack-years), median (range) & $50(4-260)$ \\
\hline \multicolumn{2}{|l|}{ Tumor Stage, n (\%) } \\
\hline $\mathrm{TO}^{\mathrm{a}}$ & $1(2)$ \\
\hline T1 & $17(33)$ \\
\hline T2 & $26(50)$ \\
\hline T3 & $8(15)$ \\
\hline \multicolumn{2}{|l|}{ Nodal Stage, $\mathrm{n}(\%)$} \\
\hline No & $41(79)$ \\
\hline N1 & $11(21)$ \\
\hline \multicolumn{2}{|l|}{ Clinical Stage, n (\%) } \\
\hline IA & $14(27)$ \\
\hline IB & $10(19)$ \\
\hline IIA & $17(33)$ \\
\hline
\end{tabular}




\section{Cureus}

Ultracentral $^{\mathrm{b}}, \mathrm{n}(\%)$

Yes

No

Primary Tumor Size (cm), median (range)

$3.1(0.9-7.4)$

Primary Tumor $\geq 5 \mathrm{~cm}, \mathrm{n}$ (\%)

Baseline Oxygen Dependence, n (\%)

Surgical Candidacy, n (\%)

Unresectable

Medically Inoperable

Both Unresectable and Medically Inoperable

Declined Surgery

\section{TABLE 1: Patient and Disease Characteristics}

cm, centimeter; ECOG, Eastern Cooperative Oncology Group; n, number; NSCLC, non-small cell lung cancer; NOS, not otherwise specified.

aPatient with N1 nodal involvement without evidence of a primary tumor.

bPTV contacts proximal bronchial tree, trachea, esophagus, pulmonary vein, or pulmonary artery among 38 node-negative patients with evaluable radiotherapy treatment plans.

The most commonly utilized fractionation was $70.2 \mathrm{~Gy}$ in 26 fractions (50 of 52) and the majority of patients (90\%) were treated using 3D-CRT (Table 2). No patients received chemotherapy or immunotherapy after irradiation. Median follow-up was 43.3 months (95\% CI 30.9-NC). 


\section{Cureus}

\begin{tabular}{|c|c|}
\hline & Value $(n=52)$ \\
\hline \multicolumn{2}{|l|}{ Reason for HRT, n (\%) } \\
\hline Proximity to critical structures ${ }^{*}$ & $26(50)$ \\
\hline Ineligible for other therapies & $12(23$ \\
\hline Size of target & $14(27)$ \\
\hline \multicolumn{2}{|l|}{ Dose / Number of Fractions, $n(\%)$} \\
\hline 70 Gy / 26 & $50(96)$ \\
\hline 68 Gy / 25 & 1 (2) \\
\hline 65 Gy / 26 & 1 (2) \\
\hline \multicolumn{2}{|l|}{ Modality, n (\%) } \\
\hline 3D-CRT & $47(90)$ \\
\hline IMRT/NMAT & $5(10)$ \\
\hline PTV Volume $\left(\mathrm{cm}^{3}\right)$, median (range) & $103.3(14.2-594.7)$ \\
\hline \multicolumn{2}{|c|}{ Percent bilateral lung recelving specitled dose, median (range) } \\
\hline 5 Gy & $45(16-75)$ \\
\hline 20 Gy & $20(4-35)$ \\
\hline 30 Gy & $15(2-29)$ \\
\hline 40 Gy & $10(1-25)$ \\
\hline Mean Lung Dose (Gy), median (range) & $12.07(3.38-20.52)$ \\
\hline Esophagus maximum dose (Gy), median (range) & $32.4(4.7-75.1)$ \\
\hline
\end{tabular}

\section{TABLE 2: Treatment Characteristics}

3D-CRT, three-dimensional conformal radiotherapy; cm, centimeter; HRT, hypofractionated radiotherapy; IMRT, intensity-modulated radiotherapy; Gy, Gray; n, number; PTV, planning target volume; VMAT, volumetric modulated arc therapy

*Proximal bronchial tree, esophagus, heart/great vessels.

\section{Survival and disease control}

Median OS and PFS for the entire cohort were 39.6 months (95\% confidence interval [CI], 24.3-55.3) and 21.0 months ( $95 \%$ CI 14.1-not calculated [NC]), respectively (Figure 1). Median OS for node-positive versus nodenegative patients was 24.3 months vs. 39.6 months (log-rank $\mathrm{p}=0.65$ ) and the median PFS was 16.4 vs. 21.0 months ( $\mathrm{p}=0.43$ ), respectively. One- and three-year PFS was $75 \%$ and $45 \%$ for stage I and $63 \%$ and $45 \%$ for stage II patients. Cumulative incidence of LRF in the entire cohort at 3 years was 35\% (Figure 2). Three-year cumulative incidence of locoregional failure for stage I and II patients was $25 \%$ and $45 \%$, respectively (Gray's $\mathrm{p}=0.20$ ). Incidence of distant failure at three years was $34 \%$ overall, as seen in Figure 3; the three-year cumulative incidence of distant failure was $45 \%$ in patients with stage I and $22 \%$ in those with stage II disease $(\mathrm{p}=0.30$ ). The most common site of distant failure in stage I patients was the lung parenchyma (three patients), followed by the brain (two), liver (two), pleural effusion (two), bone (one), abdominal organs (one) and other (one). A similar pattern was observed for stage II patients, with distant failure occurring in the lung parenchyma in four patients, pleural effusion in two, brain in one, bone in one, and other in three. Node-positive patients, compared to node-negative patients, had similar three-year CI of locoregional failure ( $41 \%$ v. $33 \%, p=0.48$ ) and distant failure ( $33 \%$ vs. $35 \%, p=0.95)$. Three-year cumulative incidence of local, regional and distant failure were as follows: $25 \%, 10 \%$, and $45 \%$ in stage I patients and $19 \%, 35 \%$, and $22 \%$ in stage II patients. 


\section{Cureus}

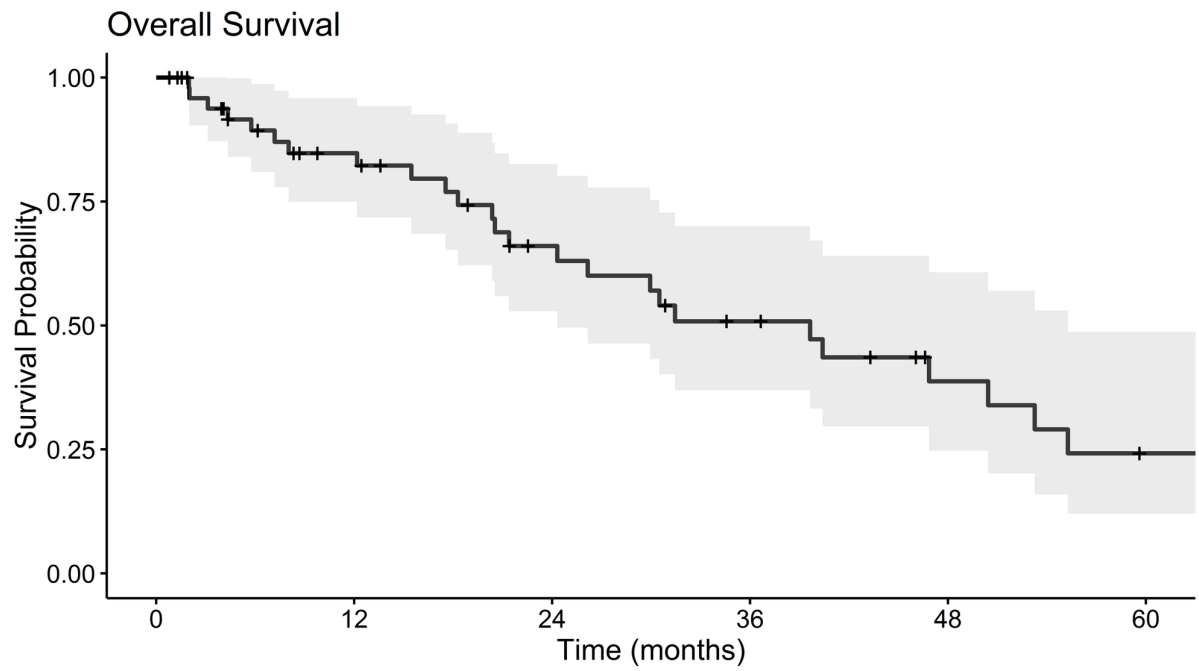

Number at risk

\begin{tabular}{|c|c|c|c|c|c|c|}
\hline All & 52 & 34 & 22 & 15 & 8 & 4 \\
\hline & 0 & 12 & 24 & 36 & 48 & 60 \\
\hline
\end{tabular}

Progression-free Survival

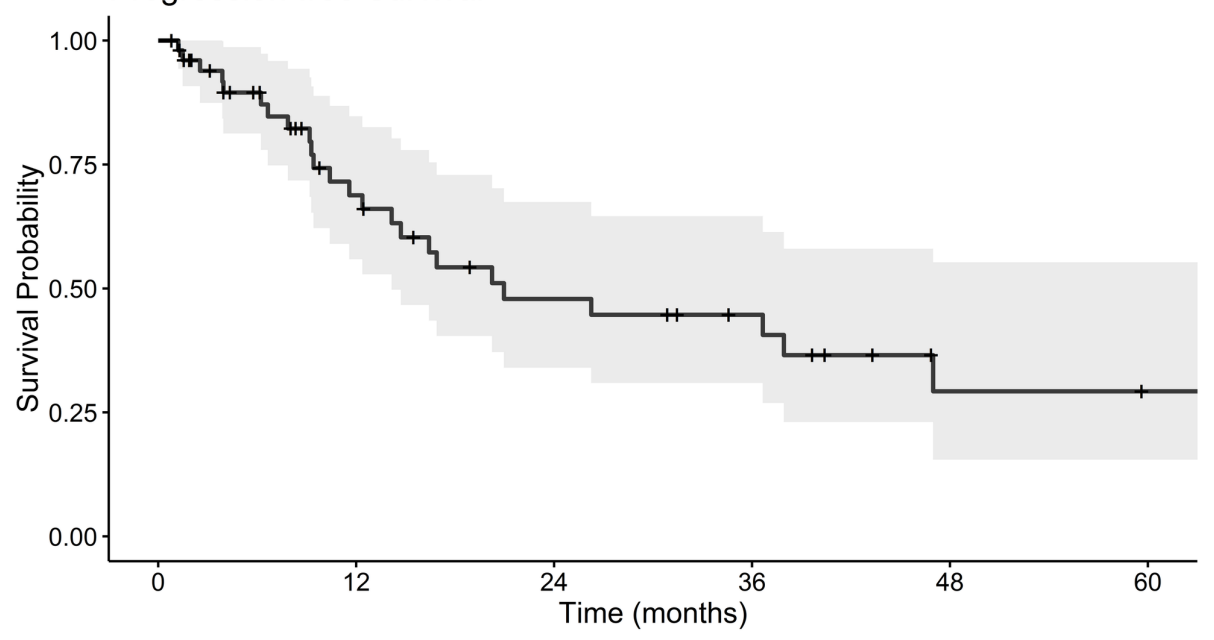

Number at risk

\begin{tabular}{|c|c|c|c|c|c|c|}
\hline \multirow{2}{*}{$\begin{array}{l}\frac{\pi}{\pi} \\
\stackrel{\pi}{\omega}\end{array}$ All } & 52 & 25 & 15 & 11 & 4 & 3 \\
\hline & 0 & 12 & & $\begin{array}{l}36 \\
\text { s) }\end{array}$ & 48 & 60 \\
\hline
\end{tabular}

FIGURE 1: Kaplan-Meier Plots of Overall Survival and Progression-free Survival 


\section{Cureus}

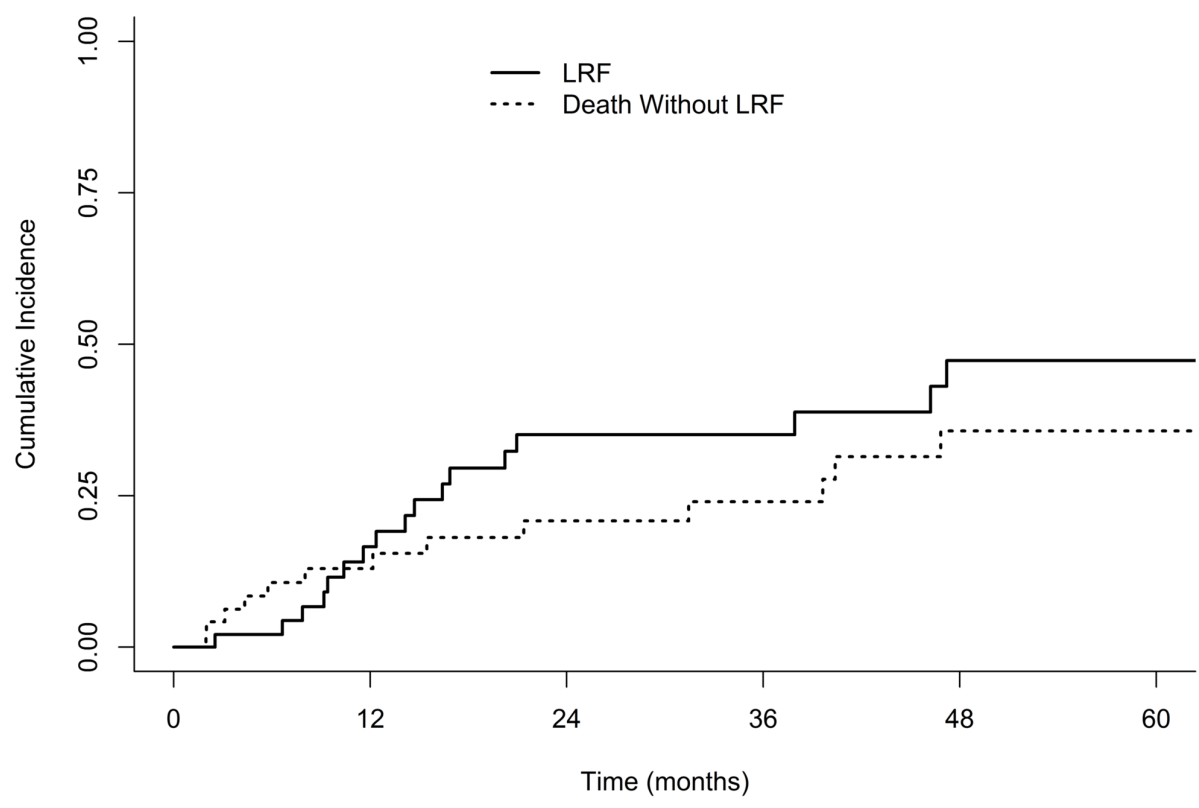

FIGURE 2: Competing-risk Cumulative Incidence of Locoregional Failure LRF, locoregional failure.

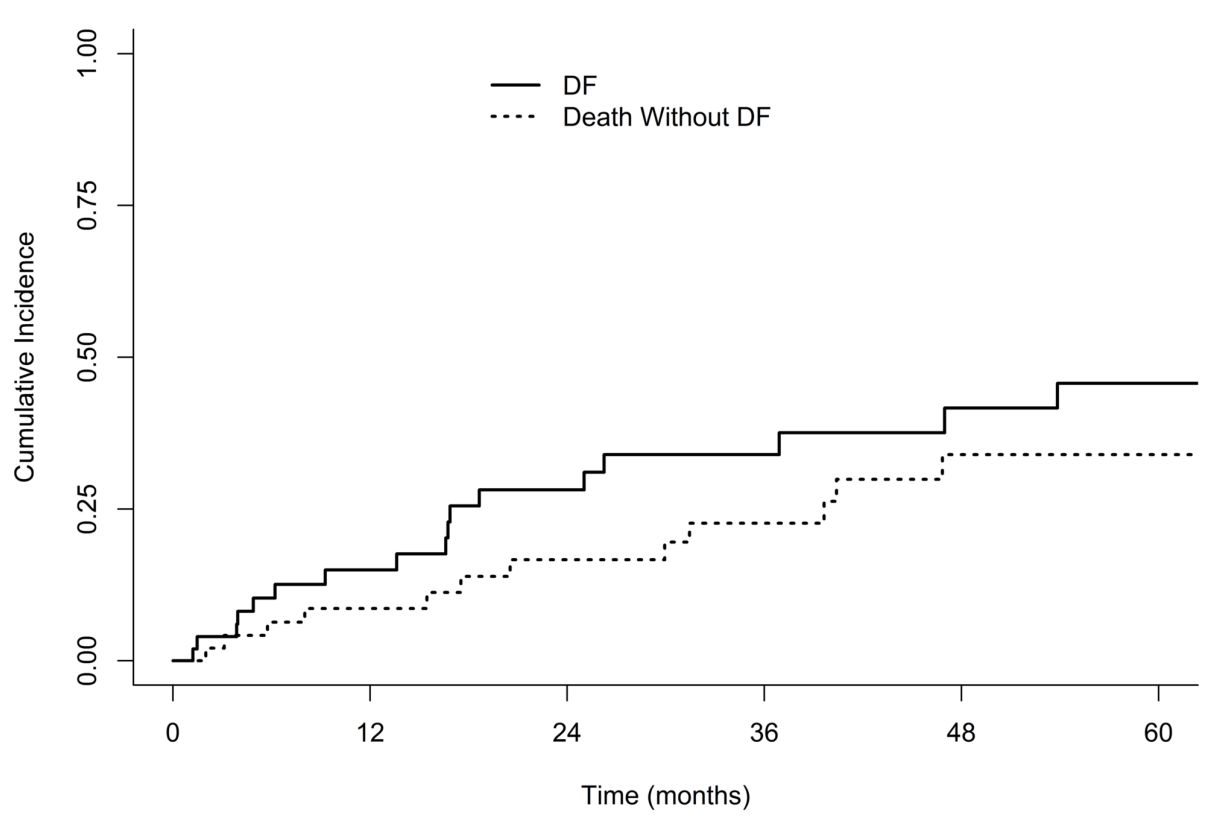

FIGURE 3: Competing-risk Cumulative Incidence of Distant Failure

DF, distant failure.

\section{Treatment-related toxicity}

Two (4\%) patients developed grade 3 pneumonitis and 9 (17\%) patients required a course of steroids for respiratory symptoms. Of the two patients with Grade 3 pneumonitis, both had ultracentral primary tumors. In these patients, the V20 of the bilateral lungs were $33 \%$ and $15 \%$. No correlation was observed between the grade of pneumonitis and bilateral lung V5, V20, V30, V40 or mean lung dose (Table 3). Bivariate logistic regression models did not identify a lung dose parameter associated with clinically significant pneumonitis (grade $\geqslant 2$ ). No patients with baseline oxygen dependence experienced grade $2+$ pneumonitis, nor did those five patients treated with intensity-modulated radiotherapy (IMRT) or volumetric modulated arc therapy (VMAT). The maximum dose to the esophagus was available in six of the eight patients experiencing acute grade 2 esophagitis; in these six patients, the median maximum esophageal dose was $67.64 \mathrm{~Gy}$ (range, 
38.34-74.40). Acute grade 2 esophagitis was associated with the maximum dose to the esophagus (OR 1.07, $95 \%$ CI 1.02-1.16, $\mathrm{p}=0.03$ ) and occurred in $9 \%$ of patients with a maximum esophageal dose $\leqslant 60$ Gy and $44 \%$ of those with doses $>60 \mathrm{~Gy}(\mathrm{p}=0.02)$. Grade 2 esophagitis was also not associated with ultracentral primary and/or node-positive disease $(\mathrm{p}=0.66)$. No patients developed late esophageal toxicity or esophageal toxicity requiring intervention such as enteral feeding tube or endoscopic dilation.

\begin{tabular}{|c|c|c|c|c|c|}
\hline & \multicolumn{4}{|c|}{ CTCAE Version 4.0 Toxicity Grade } & \multirow[b]{2}{*}{$\mathrm{p}$-value } \\
\hline & 0 & 1 & 2 & 3 & \\
\hline Pneumonitis, n (\%) & $26(50)$ & $17(33)$ & $7(13)$ & $2(4)$ & - \\
\hline \multicolumn{6}{|l|}{ Bilateral lung dose (\%), median (range) } \\
\hline 5 Gy & $45(16-75)$ & $42(35-73)$ & $45(35-56)$ & $43(35-46)$ & 0.98 \\
\hline 20 Gy & $20(4-35)$ & $22(14-33)$ & $22(14-25)$ & $24(15-33)$ & 0.35 \\
\hline 30 Gy & $15(2-29)$ & $15(8-25)$ & $18(11-28)$ & $19(10-28)$ & 0.15 \\
\hline 40 Gy & $10(1-21)$ & $10(4-19)$ & $14(8-21)$ & $16(6-25)$ & 0.27 \\
\hline Mean Lung Dose (Gy), median (range) & $11.9(3.4-1 / .9)$ & $11.8(7.7-20.3)$ & $12.7(8.4-19.1)$ & $14.4(8.2-20.5)$ & 0.29 \\
\hline Esophagitis, n (\%) & $36(69)$ & $8(15)$ & $8(15)$ & $0(0)$ & - \\
\hline Esophagus Maximum Dose (Gy), median (range) & $29.7(11.6-75.1)$ & $14.6(4.7-65.0)$ & $67.6(38.3-74.4)$ & - & 0.20 \\
\hline
\end{tabular}

\section{TABLE 3: Treatment Toxicity by Dose to Organs at Risk}

CTCAE, Common Terminology Criteria for Adverse Events; n, number, Gy, Gray.

No Grade 4-5 adverse events were observed.

\section{Discussion}

While surgery and SBRT are accepted therapeutic options for early stage NSCLC, the role of HRT alone in patients with more advanced disease or in those who are ineligible for chemotherapy, surgery, or SBRT is unclear. The results of CALGB 33904 demonstrated that HRT is a safe and effective option for nonsurgical stage I NSCLC with tumors $\leqslant 4 \mathrm{~cm}$, but data are scarce regarding patients that do not meet these criteria [9]. Our data not only confirm the tolerability and reasonable efficacy of HRT in early stage disease, but also illustrate promising outcomes for tumors unsuitable for SBRT and in patients with limited nodal disease unable to receive chemotherapy.

The median OS for the cohort of stage I-IIB patients was 39.6 months-similar to the 38.5 months reported from the CALGB study for stage IA/IB patients. Among stage I patients, the three-year LF was $25 \%$, which is less than what would be expected after SBRT [1-2,23], but higher than other HRT regimens described in the literature [17-18]. While multiple studies have noted improved tumor control with a BED10 >100 Gy using hypofractionated regimens, variation in statistical methods, dose prescription and delivery, treatment schedule, and definitions of recurrence limit the generalizability of this conclusion. In this analysis, the cumulative incidence of distant failure was $34 \%$ overall. This estimate was numerically, but not statistically significantly, higher for stage I patients compared to stage II patients. This may be due to the limited sample size. It may also be due to a higher competing risk of death in stage II patients, as more stage I patients would survive to have a local and/or distant failure event. This may also be reflective of the propensity for subsequent neoplasms in patients with a heavy smoking history. All patients in this cohort were either current or former smokers and the median smoking history was 50 pack-years, and the most common site of distant failure was the lung parenchyma for both groups.

Other recent studies have assessed the toxicity of SBRT with regard to central and ultracentral tumors $[5,8,24]$. In a series reviewing 65 patients with ultracentral tumors treated with SBRT in $\leqslant 8$ fractions, Grade $3+$ toxicity occurred in $19 \%$, including 7 (11\%) patients who experienced grade 5 toxicity [24]. In comparison, our reported fractionation was well tolerated: severe (CTCAE Grade $3+$ ) toxicity was infrequent in this cohort primarily composed of patients with ultracentral or node-positive disease. Conclusions regarding lung dosimetric predictors were limited due to the low number of pneumonitis events. With regard to esophagitis, attempts should be made to limit the maximum dose to less than $60 \mathrm{~Gy}$ based upon our observation. However, in the node-positive and ultracentral population, this may not be feasible. In the RTOG 0617 phase III clinical trial, the primary esophageal dose constraint was a mean dose of $<34 \mathrm{~Gy}$. The maximum dose to the esophagus was recorded per protocol, but to our knowledge, analyses of esophagitis by 
maximum esophageal dose have not been reported. By comparison, in a prospective, phase I dose-escalation study of accelerated HRT to $60 \mathrm{~Gy}$ with concurrent chemotherapy, the esophagus was constrained to a maximum less than $105 \%$ of the prescription dose $(63 \mathrm{~Gy})$ and the proportion of the esophageal volume receiving $>55$ Gy was constrained to $<30 \%$ [14]. In this report, our data support the assertion that HRT to 70.2 Gy is a safe and effective alternative with a reasonably low risk of grade 1-2 esophagitis.

One potential limitation to this regimen, in comparison with other regimens utilized for NSCLC not suitable for SBRT, is the patient inconvenience associated with 26 daily fractions. A Canadian, multi-institutional phase II trial included patients with T1-3N0 NSCLC that were ineligible for surgical resection due to underlying comorbidity or declined surgery [25]. HRT was delivered to a dose of $60 \mathrm{~Gy}$ in 15 fractions (BED10 $=84 \mathrm{~Gy}$ ) using 3D-conformal radiotherapy (3D-CRT), resulting in favorable primary tumor control ( $87 \%$ at two years) and low rates of toxicity (10\% grade 3-4 pneumonitis). Of note, this study excluded patients with hilar nodal involvement, primary tumors greater than $5 \mathrm{~cm}$, centrally located T3 tumors, and patients with poor performance status $(E C O G>2$ ). In the current series, $21 \%$ of patients had N1 disease, nine of 52 had primary tumors $>5 \mathrm{~cm}$, and $15 \%$ had an ECOG performance status of 3 , and yet the local control was comparable and the observed toxicity rates were slightly lower. This may be related to the difficulty of toxicity assessment via retrospective chart review, which likely underestimates these rates.

Although previous studies have described regimens that appear safe and efficacious for centrally-located, node-negative disease $[6,26]$, data for HRT alone in the management of stage IIB disease with hilar involvement are lacking. A dose-escalation study assigned patients with stage II-IV NSCLC, poor performance status (ECOG 2 or greater) who were not candidates for resection, SBRT, or concurrent chemoradiotherapy to 50,55 , or $60 \mathrm{~Gy}$ in 15 fractions [27]. None of the dose levels exceeded the maximum tolerated dose and there were few grade $\geqslant 3$ toxicities. No differences in survival were noted between groups, and local failure data were not reported due to poor survival. Dose-escalation to this extent (maximum dose level BED10 = $84 \mathrm{~Gy}$ ) as reported in this study requires highly conformal techniques such as intensity modulated radiotherapy; the majority (90\%) of patients in this study were treated using 3D conformal techniques without an increase in treatment toxicity. Additionally, the current series is comprised of patients with node-negative or limited (hilar) nodal disease with better performance status. In these patients, the risk of locoregional failure may outweigh the competing risk of death from other causes, warranting attempts to achieve a dose adequate for durable tumor control. A retrospective analysis [28] of stage II-III patients treated with HRT alone ( $93 \%$ to a dose of $66 \mathrm{~Gy}$ in 30 fractions, BED10 = 80.52 Gy) reported comparable survival in stage II patients (median OS 24 months), but two patients could not tolerate completion of RT and 59\% experienced grade 2 esophagitis. No patients in the current series failed to complete the prescribed course of radiotherapy. It is our position that this fractionation scheme represents an acceptable mildly hypofractionated alternative for hilar node-positive patients that may be delivered with routine 3D-CRT. While SBRT or similar hypofractionated regimens may be appropriate for patients with node-negative, central and/or large NSCLC [29], a more protracted regimen may be appropriate for cases where normal tissue constraints are unable to be met using such fractionation. In these cases, in addition to those with hilar involvement, 70.2 Gy in 26 fractions appears efficacious and safe.

The addition of consolidative immunotherapy has recently been shown to improve survival after chemoradiotherapy in stage III NSCLC [30]. Investigations of the role for immunotherapy after definitive SBRT for early stage NSCLC are also planned. In light of these advances, immunotherapy after definitive HRT, as is being studied in similar cohorts after surgical resection (NCT02504372, NCT02273375), may further improve outcomes.

This study is limited by its retrospective nature with the inherent selection biases therein. In addition, limitations exist regarding the detection of toxicity events by review of the electronic medical record, and there exists a small risk of underestimation of these toxicity rates. The study is limited by its small sample size, reducing the power of statistical comparisons within this group. The long duration of the study period may also be problematic with regard to changes in practice patterns in the use of SBRT and HRT for central/ultracentral NSCLC that have shifted over time. As a result, these conclusions must be limited to hypothesis generation. It should be noted that treatment options are limited for this patient population not eligible for other standard of care therapies due to centrally-located disease, inoperable and/or unresectable disease or medical comorbidity precluding SBRT or surgery. Further study into similarly hypofractionated regimens is warranted to improve local control and reduce treatment time.

\section{Conclusions}

Hypofractionated radiotherapy alone to $70.2 \mathrm{~Gy}$ is a safe and efficacious alternative for patients with stage IIIB NSCLC who are ineligible for other standard of care interventions such as surgical resection or SBRT. This regimen results in good locoregional control, comparable survival outcomes, and an acceptable toxicity profile. Further prospective study is warranted.

\section{Additional Information \\ Disclosures}


Human subjects: Consent was obtained by all participants in this study. Wake Forest University Health Science IRB issued approval IRB00046427. This study was approved by the Institutional Review Board of Wake Forest School of Medicine (IRB00046427). Animal subjects: All authors have confirmed that this study did not involve animal subjects or tissue. Conflicts of interest: In compliance with the ICMJE uniform disclosure form, all authors declare the following: Payment/services info: All authors have declared that no financial support was received from any organization for the submitted work. Financial relationships: All authors have declared that they have no financial relationships at present or within the previous three years with any organizations that might have an interest in the submitted work. Other relationships: All authors have declared that there are no other relationships or activities that could appear to have influenced the submitted work.

\section{Acknowledgements}

We would like to acknowledge the database management assistance of the Wake Forest Clinical and Translational Science Institute (WF CTSI), which is supported by the National Center for Advancing Translational Sciences (NCATS), National Institutes of Health, through Grant Award Number UL1TR001420.

\section{References}

1. Timmerman R, Paulus R, Galvin J, et al.: Stereotactic body radiation therapy for inoperable early stage lung cancer. JAMA. 2010, 303:1070-1076. 10.1001/jama.2010.261

2. Timmerman RD, Paulus R, Pass HI, et al.: Stereotactic body radiation therapy for operable early-stage lung cancer. JAMA Oncol. 2018, 4:1263-1266. 10.1001/jamaoncol.2018.1251

3. Tekatli H, van't Hof S, Nossent EJ, Dahele M, Verbakel WFAR, Slotman BJ, Senan S: Use of stereotactic ablative radiotherapy (SABR) in non-small cell lung cancer measuring more than $5 \mathrm{~cm}$. J Thorac Oncol. 2017, 12:974-982. 10.1016/j.jtho.2017.02.021

4. Timmerman R, McGarry R, Yiannoutsos C, et al.: Excessive toxicity when treating central tumors in a phase II study of stereotactic body radiation therapy for medically inoperable early-stage lung cancer. J Clin Oncol. 2006, 24:4833-4839. 10.1200/JCO.2006.07.5937

5. Tekatli H, Haasbeek N, Dahele M, et al.: Outcomes of hypofractionated high-dose radiotherapy in poor-risk patients with “ultracentral” non-small cell lung cancer. J Thorac Oncol. 2016, 11:1081-1089. 10.1016/j.jtho.2016.03.008

6. Haasbeek CJA, Lagerwaard FJ, Slotman BJ, Senan S: Outcomes of stereotactic ablative radiotherapy for centrally located early-stage lung cancer. J Thorac Oncol. 2011, 6:2036-2043. 10.1097/JTO.0b013e31822e71d8

7. Leow JJ, Martin-Doyle W, Fay AP, Choueiri TK, Chang SL, Bellmunt J: A systematic review and metaanalysis of adjuvant and neoadjuvant chemotherapy for upper tract urothelial carcinoma. Eur Urol. 2014, 66:529-541. 10.1016/j.eururo.2014.03.003

8. Raman S, Yau V, Pineda S, et al.: Ultracentral tumors treated with stereotactic body radiotherapy: singleinstitution experience. Clin Lung Cancer. 2018, 19:803-810. 10.1016/j.cllc.2018.06.001

9. Bogart JA, Hodgson L, Seagren SL, et al.: Phase I study of accelerated conformal radiotherapy for stage I non-small-cell lung cancer in patients with pulmonary dysfunction: CALGB 39904. J Clin Oncol. 2010, 28:202-206. 10.1200/JCO.2009.25.0753

10. Rodrigues G, Choy H, Bradley J, et al.: Definitive radiation therapy in locally advanced non-small cell lung cancer: Executive summary of an American Society for Radiation Oncology (ASTRO) evidence-based clinical practice guideline. Pract Radiat Oncol. 2015, 5:141-148. 10.1016/j.prro.2015.02.012

11. Senthi S, Haasbeek CJA, Slotman BJ, Senan S: Outcomes of stereotactic ablative radiotherapy for central lung tumours: a systematic review. Radiother Oncol. 2013, 106:276-282. 10.1016/j.radonc.2013.01.004

12. Kestin L, Grills I, Guckenberger M, et al.: Dose-response relationship with clinical outcome for lung stereotactic body radiotherapy (SBRT) delivered via online image guidance. Radiother Oncol. 2014, 110:499504. 10.1016/j.radonc.2014.02.002

13. Kaster TS, Yaremko B, Palma DA, Rodrigues GB: Radical-intent hypofractionated radiotherapy for locally advanced non-small-cell lung cancer: a systematic review of the literature. Clin Lung Cancer. 2015, 16:7179. 10.1016/j.cllc.2014.08.002

14. Urbanic JJ, Wang X, Bogart JA, et al.: Phase 1 study of accelerated hypofractionated radiation therapy with concurrent chemotherapy for stage III non-small cell lung cancer: CALGB 31102 (Alliance). Int J Radiat Oncol Biol Phys. 2018, 101:177-185. 10.1016/j.ijrobp.2018.01.046

15. Non-small cell lung cancer. (2019). Accessed: May 29, 2019: https://www.nccn.org/professionals/physician_gls/pdf/nscl.pdf.

16. Mauguen A, Le Péchoux C, Saunders MI, et al.: Hyperfractionated or accelerated radiotherapy in lung cancer: an individual patient data meta-analysis. J Clin Oncol. 2012, 30:2788-2797. 10.1200/JCO.2012.41.6677

17. Amini A, Lin SH, Wei C, Allen P, Cox JD, Komaki R: Accelerated hypofractionated radiation therapy compared to conventionally fractionated radiation therapy for the treatment of inoperable non-small cell lung cancer. Radiat Oncol. 2012, 7:33. 10.1186/1748-717X-7-33

18. Nguyen LN, Komaki R, Allen P, Schea RA, Milas L: Effectiveness of accelerated radiotherapy for patients with inoperable non-small cell lung cancer (NSCLC) and borderline prognostic factors without distant metastasis: a retrospective review. Int J Radiat Oncol Biol Phys. 1999, 44:1053-1056. 10.1016/S03603016(99)00130-3

19. Lucas JT Jr., Kuremsky JG, Soike M, et al.: Comparison of accelerated hypofractionation and stereotactic body radiotherapy for Stage 1 and node negative Stage 2 non-small cell lung cancer (NSCLC). Lung Cancer. 2014, 85:59-65. 10.1016/j.lungcan.2014.04.003

20. Harris PA, Taylor R, Thielke R, Payne J, Gonzalez N, Conde JG: Research electronic data capture (REDCap)-A 
metadata-driven methodology and workflow process for providing translational research informatics support. J Biomed Inform. 2009, 42:377-381. 10.1016/j.jbi.2008.08.010

21. Kong FM, Ritter T, Quint DJ, et al.: Consideration of dose limits for organs at risk of thoracic radiotherapy: atlas for lung, proximal bronchial tree, esophagus, spinal cord, ribs, and brachial plexus. Int J Radiat Oncol Biol Phys. 2011, 81:1442-1457. 10.1016/j.ijrobp.2010.07.1977

22. Common Terminology Criteria for Adverse Events (CTCAE) Version 4.0 . (2009). Accessed: May 29, 2019 : https://www.eortc.be/services/doc/ctc/CTCAE_4.03_2010-06-14_QuickReference_5x7.pdf.

23. Fakiris AJ, McGarry RC, Yiannoutsos CT, Papiez L, Williams M, Henderson MA, Timmerman R: Stereotactic body radiation therapy for early-stage non-small-cell lung carcinoma: four-year results of a prospective phase II study. Int J Radiat Oncol Biol Phys. 2009, 75:677-682. 10.1016/j.ijrobp.2008.11.042

24. Wang C, Sidiqi BU, Yorke ED, et al.: Toxicity and local control in "ultra-central" lung tumors treated with stereotactic body radiation therapy (SBRT). Int J Radiat Oncol Biol Phys. 2018, 102:10. 10.1016/j.ijrobp.2018.06.118

25. Cheung P, Faria S, Ahmed S, et al.: Phase II study of accelerated hypofractionated three-dimensional conformal radiotherapy for Stage T1-3 N0 M0 non-small cell lung cancer: NCIC CTG BR.25. J Natl Cancer Inst. 2014, 106:8. 10.1093/jnci/dju164

26. Chang JY, Li Q-Q, Xu Q-Y, et al.: Stereotactic ablative radiation therapy for centrally located early stage or isolated parenchymal recurrences of non-small cell lung cancer: how to fly in a "no fly zone". Int J Radiat Oncol Biol Phys. 2014, 88:1120-1128. 10.1016/j.ijrobp.2014.01.022

27. Westover KD, Loo BW Jr, Gerber DE, et al.: Precision hypofractionated radiation therapy in poor performing patients with non-small cell lung cancer: phase 1 dose escalation trial. Int J Radiat Oncol Biol Phys. 2015, 93:72-81. 10.1016/j.ijrobp.2015.05.004

28. Joo JH, Song SY, Kim SS, Jeong Y, Jeong SY, Choi W, Choi EK: Definitive radiotherapy alone over 60 Gy for patients unfit for combined treatment to stage II-III non-small cell lung cancer: retrospective analysis. Radiation Oncology. 2015, 10:8. 10.1186/s13014-015-0560-z

29. Verma V, Shostrom VK, Kumar SS, et al.: Multi-institutional experience of stereotactic body radiotherapy for large ( $\geqslant 5$ centimeters) non-small cell lung tumors. Cancer. 2017, 123:688-696. 10.1002/cncr.30375

30. Antonia SJ, Villegas A, Daniel D, et al.: Overall survival with durvalumab after chemoradiotherapy in stage III NSCLC. N Eng J Med. 2018, 379:2342-2350. 10.1056/NEJMoa1809697 